\title{
Finite element analysis of a Piezoelectric layered plate with different materials
}

\author{
Hossein Alimohammadi, Behrooz Izadi Babokani
}

\begin{abstract}
The objective of this study is a static analysis of smart piezoelectric ceramic plane which has infinite length and width and constant thickness. This plate has been studied by six various piezoelectric materials, the polarization direction assumed towards the third direction and loading was based on applying the stress on the upper and lower sides of the plate. Also, the analysis was conducted in both open and short circuits state according to the laws of nature. Two commercial software Abaqus and Ansys were used for the analysis, and the results were compared for all situations and materials. The results showed that the mechanical and electrical quantities had not been affected by the thickness of the piezoelectric ceramic plane, and among the piezoelectric materials, PZT5H material has the most electromechanical dependency.
\end{abstract}

Index Terms - Smart Structures, piezoelectric ceramic plane, Finite Element, Abaqus software

\section{INTRODUCTION}

The end of the twentieth century was a period of combining several branches of engineering courses that eventually could lead to change revolutionary philosophy of engineering design and created intelligence in soulless structures. The clash and the confluence of structural engineering, materials engineering, sensor systems, and measurement systems, running and energy importer systems, active and semi-active control system and artificial training networks for presence in a variety of structures. View of smart structures and materials is one of the most modern fields of structural and earthquake engineering that have been proposed to reduce the vibrations of civil engineering structures [1]. It is a combination of three fields of structural, computer and control engineering. Its Latin term is Stractronic equivalent to Mechatronics' degree in Mechanical Engineering and is based on the same philosophy. One of the most important practical applications of this nascent technology is reducing structural vibrations of mechanical, civil and aerospace engineering due to a variety of dynamic impulses, such as shaking of translational and rotational movements of the earth and the seafloor (to the earthquakes and seaquakes) [2]. Other important applications of this technology include reducing noise from the structure, controlling and reducing the vibration, active controlling the shape and geometry of the rail structures, automatic pathology, automatic monitoring of the health and life of active surveys of the rail structures [3].

Piezoelectric materials are used for two main purposes [4]: (1) measurement of noise signals (earthquake and wind), signals of the control system and all signals including

Hossein Alimohammadi, M.SC. in Earthquake Engineering and currently is Geotechnical engineering Ph.D. student in Iowa State University, Ames, Iowa, United States of America. information of the system and the environment in general Measuring tool in control is the so-called sensor or sensors, including piezopolymers like PVDF, (2) actuators and power importers or triggers for applying neutral force, the effect of turbulence in the environment and the piezoceramics such as PZT. From combining the sensors, actuators, microprocessors and microcontrollers and reasonable connections and to host structure as a host and using control logic system automation, a control circuit feedback loop is produced which is used for smart host structures [5]. In the structural laws, piezoelectric materials were established between mechanical quantities (tensors of stress and strain) and electrical quantities (vectors of the electric field strength and power flux density)[6] there is a single connection. In this way, the strain caused by the mechanical loading on the piezoelectric material, producing electric flux and eventually creating an electrical current through the material (property of direct piezoelectric sensor) is in the way which applying an electric field is induced with intensity determined by the production of stresses (inverse piezoelectric effect or property of applying) [7].

\section{ANALYTICAL SOLUTION METHOD}

The problem is solved by helping the laws of nature, laws governing the structure of piezoelectric materials and mechanical and electrical boundary conditions. The plane is shown in Fig. 1 [8].

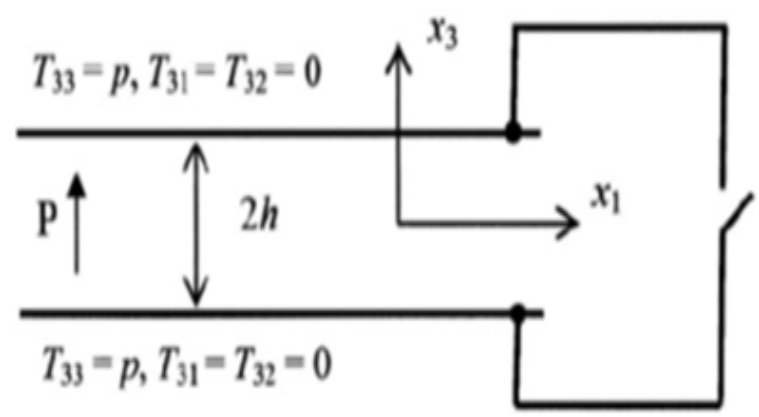

Fig.1 Electrode-graded ceramic plate under mechanical loads [8]

Before solving the problem, some symptoms used in the formulas are described: $\mathrm{C}_{\mathrm{ijk}}$ fourth-order elastic modulus tensor, $\mathrm{D}_{\mathrm{i}}$ vector of electric flux density, $\mathrm{e}_{\mathrm{ijkl}}$ third-order tensor of piezoelectric stress factors, $E_{i}$ vector of electric field intensity, Sij linear Lagrange strain tensor, $\mathrm{T}_{\mathrm{ij}}$ Cauchy stress tensor, $\varepsilon_{\mathrm{ij}}$ second-order tensor of Electricity coefficients, $\varphi_{\mathrm{i}}$ electrical potential, it is also necessary to mention the values and characteristics of the materials tested in Tables 1 and 2: 
Table.1 Tensor-tensile coefficients of piezoelectric materials

\begin{tabular}{|l|l|l|l|l|l|l|}
\hline Materials & $c_{11}$ & $c_{12}$ & $c_{13}$ & $c_{33}$ & $c_{44}$ & $c_{66}$ \\
\hline PZT 4 & 13.9 & 7.78 & 7.40 & 11.5 & 2.56 & 3.06 \\
\hline PZT 5A & 12.1 & 7.59 & 7.54 & 11.1 & 2.11 & 2.26 \\
\hline PZT 6B & 16.8 & 8.47 & 8.42 & 16.3 & 3.55 & 4.17 \\
\hline PZT 7A & 14.8 & 7.61 & 8.13 & 13.1 & 2.53 & 3.60 \\
\hline PZT 8 & 13.7 & 6.99 & 7.11 & 12.3 & 3.13 & 3.36 \\
\hline PZT 5H & 12.6 & 7.95 & 8.41 & 11.7 & 2.3 & 2.35 \\
\hline Units & $\times 10^{-8}(C / \mathrm{Vm})$ & $\times 10^{-8}(\mathrm{C} / \mathrm{Vm})$ \\
\hline
\end{tabular}

Table.2 Tensor stress tensor coefficients and piezoelectricity electrical tensioning

\begin{tabular}{|l|l|l|l|l|l|}
\hline Materials & $e_{31}$ & $e_{33}$ & $e_{15}$ & $\varepsilon_{11}$ & $\varepsilon_{33}$ \\
\hline PZT 4 & -5.2 & 15.1 & 12.7 & .646 & .562 \\
\hline PZT 5A & -5.4 & 15.8 & 12.3 & .811 & .735 \\
\hline PZT 6B & -.9 & 7.1 & 4.6 & .360 & .342 \\
\hline PZT 7A & -2.1 & 9.5 & 9.2 & .407 & .208 \\
\hline PZT 8 & -4 & 13.2 & 10.4 & .797 & .514 \\
\hline PZT 5H & -6.5 & 23.2 & 17 & 1.505 & 1.302 \\
\hline Units & $\left(C / m^{2}\right)$ & & $\times 10^{-8}(C / N m)$ \\
\hline
\end{tabular}

Conditions governing the issue are as follows:

$$
\begin{array}{lc}
T_{j i, j}=0 & D_{i, i}=0 \\
T_{i j}=c_{i j k l}^{E} S_{k l}-e_{k i j} E_{k}, & D_{i}=e_{i k l} S_{k l}+\varepsilon_{i k}^{S} E_{k} \\
S_{i j}=\frac{1}{2}\left(u_{i, j}+u_{j, i}\right), & E_{i}=-\varphi_{i} \\
T_{j i} n_{j}=p \delta_{3 i}, & x_{3}={ }_{-}^{+}{ }_{h} \\
\varphi\left(x_{3}=h\right)=\varphi\left(x_{3}=-h\right) & \\
D_{3}\left(x={ }_{-}^{+} h\right)=0 &
\end{array}
$$

The amount of tension along axis three is assumed to equal $\left(\mathrm{T}_{33}=\mathrm{p}=1000 \mathrm{~N} / \mathrm{m}^{2}\right.$ ) Relations (1), (2), and (3) are true in-plane volume. Top draft $\mathrm{E}$ in equation $\mathrm{C}^{\mathrm{E}}{ }_{\mathrm{ijkl}}$ means that the fourth-order elasticity tensor of the electric field $\mathrm{E}$ is constant and the top draft of $S$ in equation $\varepsilon{ }^{\mathrm{s}}{ }_{\mathrm{ij}}$, namely second-order tensor electricity is constant under mechanical strain. Given that the stress applied, and polarizations are applied in direction three. Displacement and electrical potential fields are assumed in direction three as follows:

$u_{3}=u_{3}\left(x_{3}\right), u_{1}=u_{2}=0, \varphi=\varphi\left(x_{3}\right)$

Relations between mechanical (stress and strain), electrical quantities (electric field intensity, electric flux density) in piezoelectric materials (trans-trophic) are as follows:
$T_{11}=c_{11}^{E} u_{1,1}+c_{12}^{E} u_{2,2}+c_{13}^{E} u_{3,3}+e_{31} \varphi_{3}$

$T_{22}=c_{12}^{E} u_{1,1}+c_{11}^{E} u_{2,2}+c_{13}^{E} u_{3,3}+e_{31}^{\varphi} \varphi_{, 3}$

$T_{33}=c_{13}^{E} u_{1,1}+c_{13}^{E} u_{2,2}+c_{33}^{E} u_{3,3}+e_{33} \varphi_{, 3}$

$T_{23}=c_{44}^{E}\left(u_{2,3}+u_{3,2}\right)+e_{15} \varphi_{, 2}$

$T_{13}=c_{44}^{E}\left(u_{1,3}+u_{3,1}\right)+e_{15} \varphi_{, 1}$

$T_{12}=c_{66}^{E}\left(u_{1,2}+u_{2,1}\right)$

$D_{1}=e_{15}\left(u_{1,3}+u_{3,1}\right)-\varepsilon_{11}^{S} \varphi_{, 1}$

$D_{2}=e_{15}\left(u_{2,3}+u_{3,2}\right)-\varepsilon_{11}^{S} \varphi_{, 2}$

$D_{3}=e_{13}\left(u_{1,1}+u_{2,2}\right)+e_{33} u_{3,3}-\varepsilon_{33}^{S} \varphi_{, 3}$

Non-zero components of the strain tensor, vector of electric field intensity, electric flux, and stress tensor are as follows:

$S_{33}=u_{3,3}$

$E_{3}=-\varphi_{3}$

$T_{11}=T_{22}=c_{13}^{E} u_{3,3}+e_{31} \varphi_{, 3}$

$T_{33}=c_{33}^{E} u_{3,3}+e_{33} \varphi_{, 3}=P$

$D_{3}=e_{33} u_{3,3}-\varepsilon_{33}^{S} \varphi_{3}$

According to relations 17 to 21 , the movement and electrical charge equations can be written as follows:

$T_{33,3}=c_{33}^{E} u_{3,33}+e_{33} \varphi_{33}=0$

$D_{3,3}=e_{33} u_{3,33}-\varepsilon_{33}^{S} \varphi_{33}=0$

So, it can be stated that:

$u_{3,33}=0 \quad \varphi_{, 33}=0$

A. A. Open circuits state

Articles in this mode of electric flux density (electric displacement) or the load at both ends of the circuit will be zero, as shown in Fig. 2 [8].

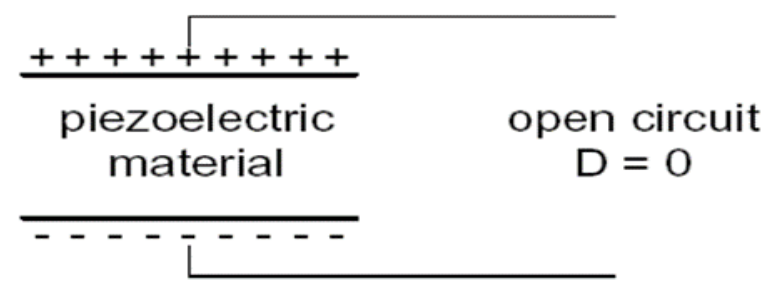

Fig.2 Open circuits state [8] 
According to the terms of mechanical we have:

$$
\begin{aligned}
& S_{3}=u_{3,3}=\frac{P}{c_{33}\left(1+k_{33}^{2}\right)} \\
& E_{3}=-\varphi_{, 3}=-\frac{e_{33}}{\varepsilon_{33} c_{33}\left(1+k_{33}^{2}\right)} P \\
& T_{11}=T_{22}=c_{13}^{E} u_{3,3}+e_{31} \varphi_{3} \\
& T_{33}=c_{33}^{E} u_{3,3}+e_{33} \varphi_{, 3}=P \\
& D_{3}=e_{33} u_{3,3}-\varepsilon_{33}^{S} \varphi_{, 3}=0
\end{aligned}
$$

The work done by the page in the unit volume in open circuit mode is as follows:

$W_{1}=\frac{1}{2} T_{33} S_{33}=\frac{P^{2}}{2 c_{33}\left(1+k_{33}^{2}\right)}$

Numerical results of stress, strain, and electric field for different materials in an open circuit condition is shown in Table 1.

The ratio $\mathrm{e}_{33}^{2} /\left(\varepsilon_{33} \mathrm{c}_{33}\right)$ is called electromechanical dependency 1 and is shown by $\mathrm{k}_{33}^{2}$. The larger the proportion, the stronger electromechanical connection will be.

The comparison of the ratio $\mathrm{k}_{33}^{2}=\mathrm{e}_{33}^{2} /\left(\varepsilon_{33} \mathrm{c}_{33}\right)$ for different materials are shown in Table 3 .

Table. 3 Comparing $\mathrm{k}_{33}^{2}$ and materials

\begin{tabular}{|l|l|}
\hline Materials & $\mathrm{K}_{33}^{2}$ \\
\hline$P Z T 4$ & 0.35 \\
\hline$P Z T 5 A$ & 0.306 \\
\hline$P Z T 6 B$ & 0.09 \\
\hline$P Z T 7 A$ & 0.33 \\
\hline$P Z T 8$ & 0.27 \\
\hline$P Z T 5 H$ & 0.36 \\
\hline
\end{tabular}

Thus, it can be concluded that the piezoelectric materials of PZT5H material have most dependent electromechanical material. That's why among the substances most commonly used one is as a sensor and actuator.

\section{B. B. Short circuits state}

If two ends of the circuit are connected, as shown in Fig. 3, there is not any potential conflict [9]

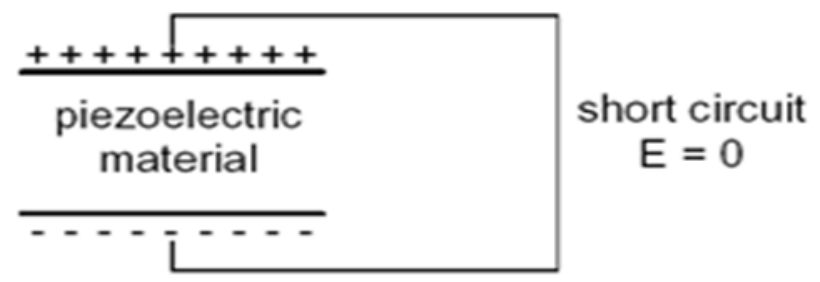

Fig. 3 Short circuits state [9]
According to the prevailing mechanical conditions, components of the stress tensor, strain tensor, vector electric flux density are as follows:

$$
\begin{aligned}
& S_{3}=u_{3,3}=\frac{P}{c_{33}} \\
& T_{11}=T_{22}=\frac{c_{13}}{c_{33}} P \\
& D_{3}=\frac{e_{33}}{c_{33}} P
\end{aligned}
$$

Work performed by plane per volume is as follows:

$W_{2}=\frac{1}{2} T_{33} S_{33}=\frac{P^{2}}{2 c_{33}}$

According to the fact that $\mathrm{k}_{33}^{2}$ is less than one of the works done in a short circuit is more than on the open circuit [10]. $W_{1}\left\langle W_{2}\right.$

Numerical results of stress-strain the electric flux density is shown in Table 2 for different materials in short circuit condition. Given that the effect of thickness was not seen in the above relationships and it can be concluded that the mechanical and (stress and strain) electric quantities (electric field intensity, electric flux density) are independent on the thickness.

\section{FINITE ELEMENT METHOD USING COMMERCIAL SOFTWARE ABAQUS AND ANSYS}

For finite element simulation, eight-node linear piezoelectric element C3D8E is used in ABAQUS Software for finite element purposes [11], and element SOLID5 is used in ANSYS Software. For modeling plane with infinite dimensions and thickness $2 \mathrm{~h}$, a plane with the dimensions of 1 $\times 1 \times 0.1$ meters is modeled. For considering the infinite conditions, the plane should consider it to be clamped on all four sides because the infinite elements in software have not piezoelectric properties. In Tables 4 and 5, the results of open circuit and in Tables 6 and seven, the short circuit results are shown.

Table.4 Comparison of numerical results in open circuit mode with Abaqus

\begin{tabular}{|l|l|l|l|}
\hline Materials & $\mathrm{S}_{33}$ & $\mathrm{E}_{3}$ & $\mathrm{~T}_{11}=\mathrm{T}_{22}$ \\
\hline$P Z T 4$ & $6.43 E-9$ & -17.3 & 385.9 \\
\hline$P Z T 5 A$ & $6.89 E-9$ & -14.8 & 440.34 \\
\hline$P Z T 6 B$ & $5.6 E-9$ & -11.68 & 461 \\
\hline$P Z T 7 A$ & $5.7 E-9$ & -26.2 & 408.4 \\
\hline$P Z T 8$ & $6.3 E-9$ & -16.44 & 387 \\
\hline$P Z T 5 H$ & $6.31 E-9$ & -11.29 & 456.44 \\
\hline
\end{tabular}




\section{CONCLUSIONS AND DISCUSSIONS}

The analysis and results of the tables in the earlier section have achieved the following results:

- The mechanical and electrical quantities have not been affected by the thickness

- Among the piezoelectric materials, PZT5H material has a most electromechanical dependency.

- The energy in short circuit is more than an open circuit, and thus the difficulty of the structure in the circuit is greater.

Table.5 Comparison of numerical results in open circuit mode with Ansys

\begin{tabular}{|l|l|l|l|}
\hline Materials & $\mathrm{S}_{33}$ & $\mathrm{E}_{3}$ & $\mathrm{~T}_{11}=\mathrm{T}_{22}$ \\
\hline$P Z T 4$ & $6.43 e-9$ & -17.3 & 383.5 \\
\hline$P Z T 5 A$ & $6.9 e-9$ & -14.8 & 437.9 \\
\hline$P Z T$ 6B & $5.6 e-9$ & -11.7 & 460.5 \\
\hline PZT 7A & $5.7 e-9$ & -26.3 & 408.7 \\
\hline PZT 8 & $6.3 e-9$ & -16.4 & 386 \\
\hline PZT 5H & $6.31 e-9$ & -11.31 & 455.44 \\
\hline
\end{tabular}

Table.6 Comparison of numerical results in short circuit mode with Abaqus

\begin{tabular}{|l|l|l|l|}
\hline Materials & $\mathrm{S}_{33}$ & $\mathrm{D}_{3}$ & $\mathrm{~T}_{11}=\mathrm{T}_{22}$ \\
\hline$P Z T 4$ & $8.69 e-9$ & $1.31 e-7$ & 643.47 \\
\hline$P Z T 5 A$ & $9 e-9$ & $1.42 e-7$ & 679.3 \\
\hline$P Z T 6 B$ & $6.13 e-9$ & $4.35 e-8$ & 516.6 \\
\hline$P Z T 7 A$ & $7.6 e-9$ & $7.25 e-8$ & 620.6 \\
\hline$P Z T 8$ & $8.13 e-9$ & $1.07 e-7$ & 578 \\
\hline$P Z T 5 H$ & $8.54 e-9$ & $1.99 e-7$ & 718.8 \\
\hline
\end{tabular}

Table.7 Comparison of numerical results in short circuit mode with Ansys

\begin{tabular}{|l|l|l|l|}
\hline Materials & $\mathrm{S}_{33}$ & $\mathrm{D}_{3}$ & $\mathrm{~T}_{11}=\mathrm{T}_{22}$ \\
\hline$P Z T 4$ & $8.7 e-9$ & $1.32 e-7$ & 640.2 \\
\hline$P Z T 5 A$ & $9.04 e-9$ & $1.43 e-7$ & 677 \\
\hline$P Z T 6 B$ & $6.13 e-9$ & $4.37 e-8$ & 514.4 \\
\hline$P Z T 7 A$ & $7.6 e-9$ & $7.3 e-8$ & 617.7 \\
\hline$P Z T 8$ & $8.13 e-9$ & $1.07 e-7$ & 574.7 \\
\hline$P Z T 5 H$ & $8.59 e-9$ & $2 e-7$ & 715.6 \\
\hline
\end{tabular}

\section{REFERENCES}

[1] J. Yang, An introduction to the theory of piezoelectricity. Springer, 2005.

[2] A. C. Eringen and G. A. Maugin, Electrodynamics of Continua I. New York, NY: Springer New York, 1990.

[3] M. Lax and D. F. Nelson, "Linear and nonlinear electrodynamics in elastic anisotropic dielectrics," Phys. Rev. B, vol. 4, no. 10, pp. 3694-3731, 1971.

[4] J. S. Yang and R. C. Batra, "Conservation laws in linear piezoelectricity,” Eng. Fract. Mech., vol. 51, no. 6, pp. 1041-1047, 1995.

[5] S. Pourianejad and B. Movahedi, "Solid-state synthesis of modified (Bi0.5Na0.5)TiO3 piezoelectric nanocrystalline ceramics and evaluating their properties," Mater. Sci. Semicond. Process., vol. 29, pp. 337-344, 2015.

[6] S. Helwany, Applied soil Mechanics with Abaqus applications. 2010.

[7] M. C. Constantinou, I. Kalpakidis, A. Filiatrault, and R. a E. Lay, "LRFD-Based Analysis and Design Procedures for Bridge Bearings and Seismic Isolators," Mceer, vol. MCEER-11-0, no. 65, pp. 1-371, 2011.

[8] E. F. Crawley and J. De Luis, "Use of piezoelectric actuators as elements of intelligent structures," AIAA J., vol. 25, no. 10, pp. 1373-1385, 1987.

[9] O. J. Aldraihem and A. A. Khdeir, "Smart beams with extension and thickness-shear piezoelectric actuators," Smart Mater. Struct., vol. 9, no. 1, pp. 1-9, Feb. 2000.

[10] IEEE standard on piezoelectricity : an American national standard New York N.Y.: Institute of Electrical and Electronics Engineers, 1988.

[11] H. Alimohammadi and M. Abu-Farsakh, "Finite Element Parametric Study on Rutting Performance of Geosynthetic Reinforced Flexible Pavements," Transp. Res. Board, vol. 98, 2019.

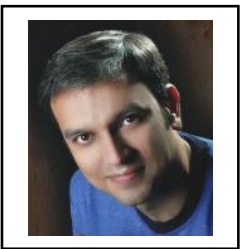

Hossein Alimohammadi has M.SC. in Earthquake Engineering and currently is Geotechnical engineering $\mathrm{Ph} . \mathrm{D}$. student in Iowa State University, Ames, Iowa, United States of America. 